\title{
FOREST FIRES IN IZMIR REGIONAL DIRECTORATE OF FORESTRY
}

\author{
KuCUKOSMANOGLU, A. ${ }^{1}-$ UZMEZ, İ. $^{2}$ \\ ${ }^{I}$ Faculty of Forestry, Istanbul University-Cerrahpasa, Istanbul, Turkey \\ ${ }^{2}$ General Directorate of Forestry, Ankara, Turkey \\ *Corresponding author \\ e-mail:aliko@istanbul.edu.tr
}

(Received $19^{\text {th }}$ Feb 2019; accepted $10^{\text {th }}$ Apr 2019)

\begin{abstract}
One of the major problems in many countries is the forest fires. Forest fires are the source of the most of the problems of fire protection organization in every year. Why does Turkey still have too many forest fires? And how do we eliminate forest fires? We are trying to approach this problem under our country conditions, and explain that how we are able to eliminate the forest fires in Izmir Forest Directorate. Forest fires stands out especially in the provinces of forests under the influence of the Mediterranean climate in Turkey. However, the forest directorate that are at risk in terms of both burning areas and fire numbers in these provinces are Mugla, Antalya and Izmir. For this reason, this study was carried out in Izmir Regional Directorate of Forestry between the years 2006-2015, one of the regional directorates that are always at risk.
\end{abstract}

Keywords: Izmir, forest fire, forest directorate, burnt area, forest conservation

\section{Introduction}

Forest fires are one of the important factors that jeopardize the continuity of forests in various parts of the world, especially in developed countries. Forest fires do not only damage timber and other forest products, they also harm ecosystem services, such as conserving the top soil etc. They destroy the habitats of forest biota and bring death to all sort of creatures. Even if the burned land is restored and reforested, forest fire change environmental conditions in forest (Mol et al., 1997). According to the recent fire statistics (from 1988 to 2016) in Turkey, the annual average number of forest fire is 2114 and that of burned forested area is 10615 ha (Goltas et al., 2017a).

Today, the importance of forest fires has increased due to global warming. Especially in the European continent, countries in the Mediterranean climate zone with low humidity and high temperature values are under the threat of forest fires with global warming. This situation is a top problem in terms of the sustainability of forests. Because of heat waves and drought, risk of forest fire, fire intensity, number of fire, fire frequency and burning area is expected to increase (Goltas et al., 2017b).

A large proportion of Turkey lies within the Mediterranean climate zone, which is characterized by hot and arid summer seasons, followed by warm and mild winters. Similar to many countries in the Mediterranean basin, Turkey is a country, which is prone to forest fires, which are frequently encountered phenomena due to climatic conditions (Mol and Kucukosmanoglu, 1998). In Turkey, about 35\% of total forested areas are sensitive to forest fires at first-degree, $23 \%$ at second-degree, and $22 \%$ at third-degree (Bilgili and Kucuk, 2001).

Therefore they should be considered as usual events. Unfortunately, forest fires are rarely a result of natural causes such as the lightning. Forest fires are mostly human 
induced events (Demir et al., 2009). Manager of the fire fighting team should comprehend the behavior of fire and customize a tailor made fire fighting strategy (Ayberk, et al., 2010).

The aim of this study is to make a detailed assessment of forest fires in general Turkey and in particular İzmir benefiting from fire reports, fire statistics, forest inventories and management plans. Results of the study will contribute to fire protection and fire suppression activity in both Turkey and Izmir Forest Directorate.

\section{Materials and methods}

The forest fires statistics occurred in Turkey between the years of 2006-2015 were evaluated in various aspects. In this study; Causes of forest fires, distribution in the field (hectare), the numerical distribution to the regional directories and the number of forest fires and total burned area in İzmir Regional Directorate of Forestry has investigated. Among the State forest Enterprises of regional Directorates; Bayındır, Bergama, İzmir, Menderes, Akhisar, Demirci, Gördes and Manisa are particularly considered. The main data of these directorates are sorted by years and calculated one by one in order to create systematic schemes. Moreover to reveal accurate investigation over these numbers, their 10 years total and 10 years average values are conceived and examined. For the investigation of forest fires in 10-year period; forest inventory and forest management plans obtained from İzmir Regional Directorate of Forestry in Izmir region has taken into consideration. In this way, the fire sensitivity of the Izmir Regional Directorate of Forestry has been tried to determine.

\section{Study area}

Surrounded by the Mediterranean, the Black Sea, the Sea of Marmara and the Aegean Sea; Turkey has $97 \%$ of its land area in Asia and 3\% in Europe. It also has $8333 \mathrm{~km}$ of coastline and related to its geographical position, properties such as climate, relief and location have caused the formation of different geographical regions within the boundaries of the country (Demir et al., 2009).

Izmir regional directorate of forestry, which includes a large part of the Aegean region, is located within the territorial boundaries of Izmir and Manisa provinces. It is surrounded by Balikesir in the north, Denizli and Kutahya in the east, Mugla Forest Regional Directorates in the south and Aegean Sea in the west. The geographical location of the Izmir Forest Directorate is located between the northern longitude of $39^{\circ} 35^{\prime} 38^{\prime \prime}-37^{\circ} 52^{\prime} 30^{\prime \prime}$ and eastern latitudes of $26^{\circ} 11^{\prime} 42^{\prime \prime}-28^{\circ} 52^{\prime} 28^{\prime \prime}$ (Fig. 1).

Under the influence of the Mediterranean climate, the plant species determined by this climate are located in İzmir. For many years, a wide variety of plant species have been destroyed due to grazing, breeding, smuggling, fire and insect damages. Especially in these areas where main tree species were damaged, drought resistant forest species were included. Main forest species are Pinus brutia and Pinus nigra in Izmir. Additionally there are many other tree species like Pinus pinea, Quercus sp., Castanea sp., Juglans sp., Fagus sp. in Izmir forests. General area of Izmir Forest Directorate is $2,509,336$ ha and $39 \%$ of this area is forest lands. This value is calculated by creating relevant pie-chart, that presents damaged forests (22\%), efficient forests (17\%) and nonforest area (61\%). 417,910 ha efficient forests, 557,095 ha damaged forest in Izmir Forest Directorate (Uzmez, 2011) (Fig. 2). 


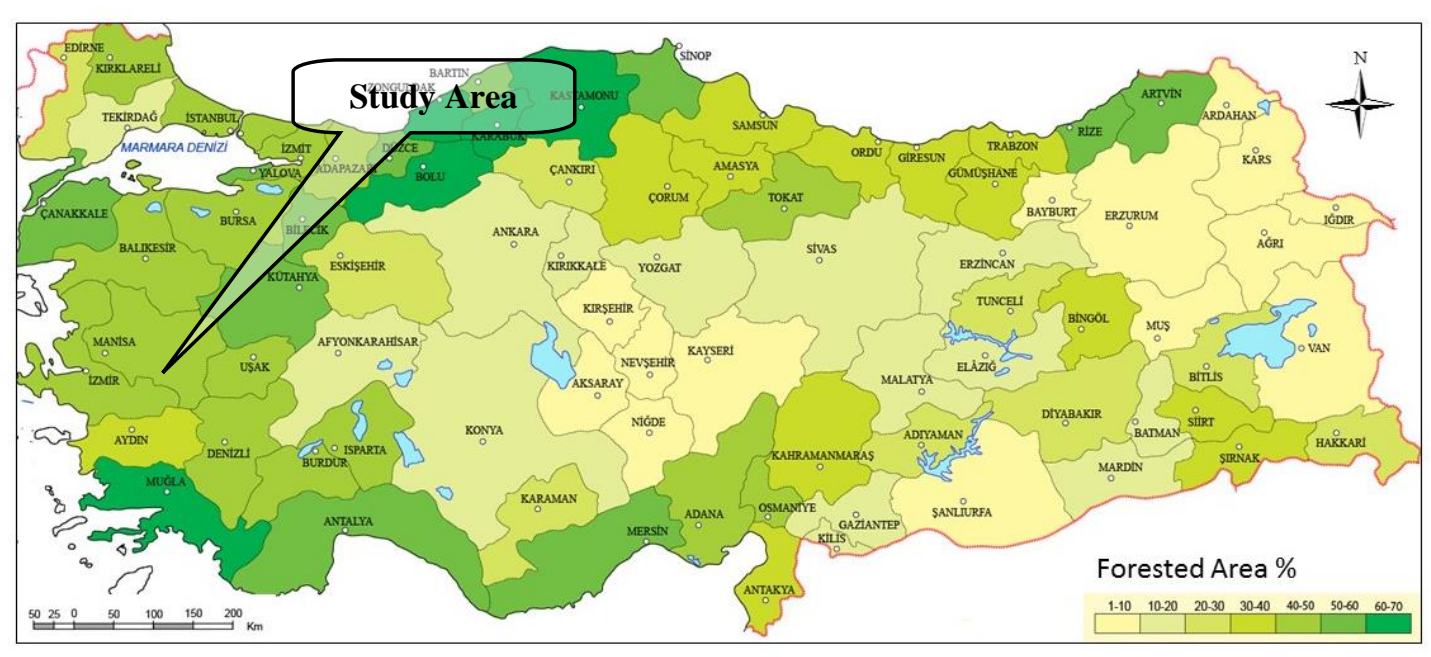

Figure 1. The location of the study area in Turkey

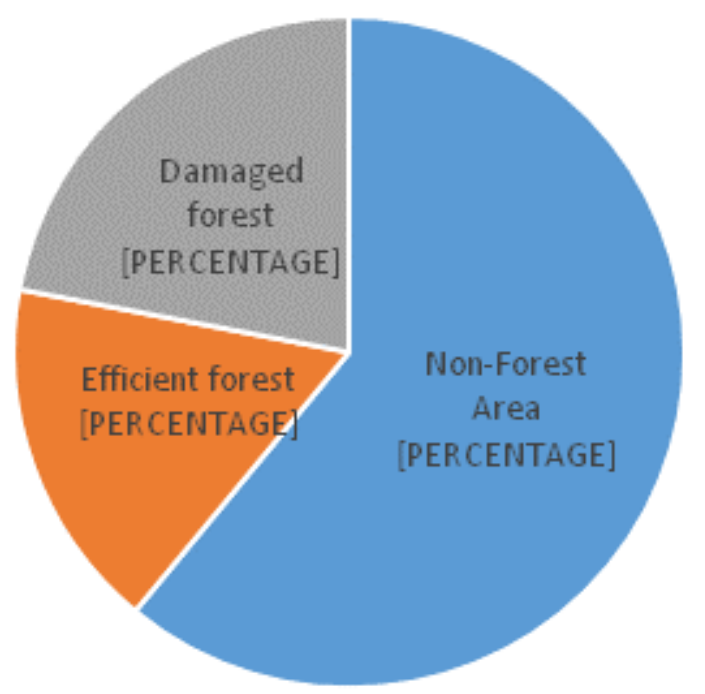

Figure 2. Forest status in İmir Forest Directorate

\section{Results}

\section{Different factors influencing forest fire danger}

Many factors such as weather conditions, forest vegetation and fire season influence the forest fire danger (Noble et al., 1980). For instance, some weather conditions such as high air temperature, low relative humidity and dry winds reduce the moisture contents of flammable materials. In Mediterranean region the wind of South, South East and South West are oceanic origin, and so they bring more humidity. As a result, the fire danger is less. But the wind of North, North East and North West origin, bring dry air masses, because they are Central Anatolian origin. So, this situation created severe fire seasons (Mol and Kucukosmanoglu, 1998).

Forest vegetation also has a great influence on the damage of the forest fires. Most of the forest fires in Turkey occur in pine forest (49\%) At the same time it is difficult to 
prepare a fire season list for different parts of Turkey, because of various climatic conditions and forest fuels. But we frequently notice almost two characteristic types of fire season. Short fire season, this is well illustrated in Black-Sea and Marmara regions. In Black-Sea region July-August, and in Marmara region June-August are the peak points. Long fire season, Mediterranean and Aegean regions are typical examples. It continues about 5-8 months, but some part of these regions has year-long fire season (Demir et al., 2009).

\section{Forest fire statistics in Turkey}

The major destructive factor in Turkish forests is forest fires. Large or small, high severity or low severity they have had a profound influences on forests and wildlands. Especially the Mediterranean area has very suitable combustion and spreading factors. Forest fires cause great damage especially in Mediterranean, Aegean and Marmara regions. Forest fires not only cause the destruction of forest resources, but also destroy the balance of the ecological system. The coastal band, which starts from Hatay in particular from the Mediterranean and the Aegean coastal regions to Istanbul, constitutes the most risky area in terms of fires. In this region, the first sensitive area of fire is 7,182,051 ha, the second sensitive area to the fire is 5,091,788 ha. According to this, 12 million ha of the forests corresponding to approximately $60 \%$ of our forests are located in areas sensitive to fire (Uzmez, 2011) (Fig. 3).

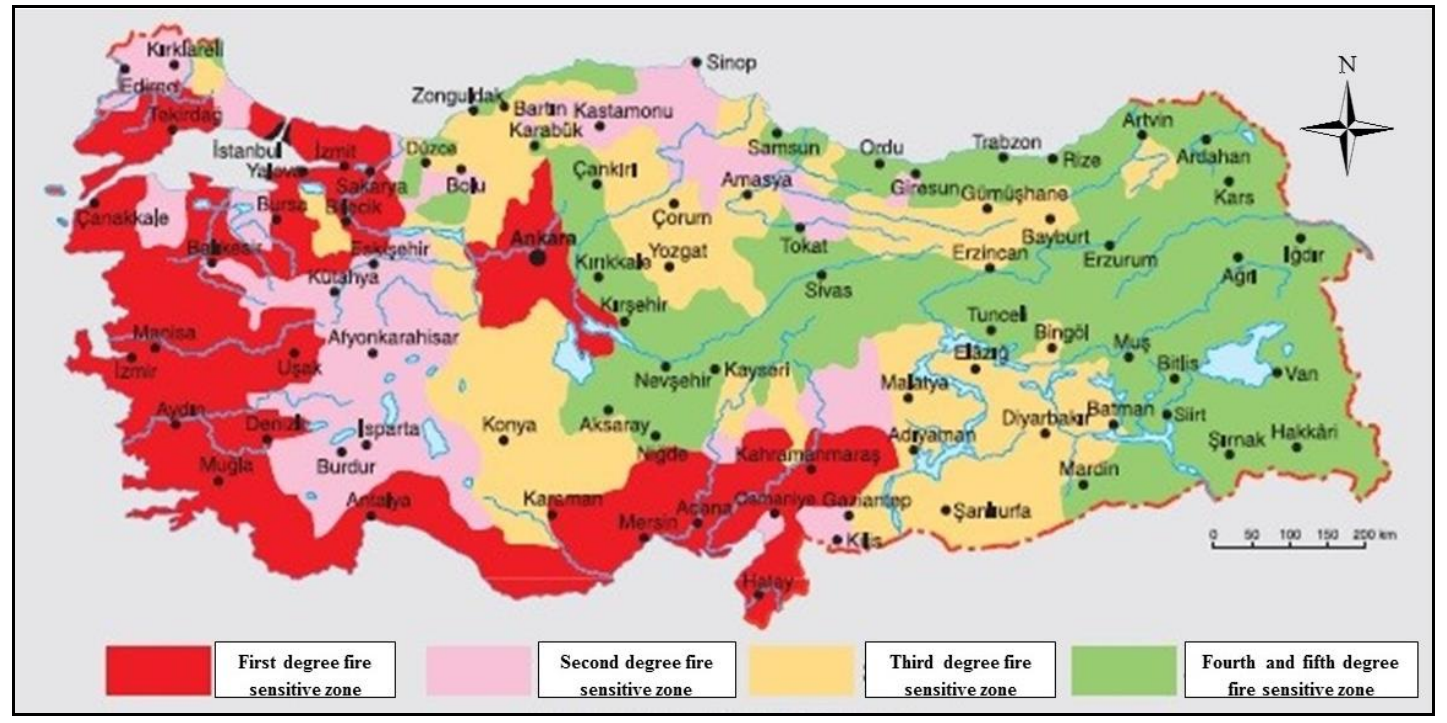

Figure 3. Sensitive regions of Turkey against to forest fire

Considering the causes of forest fires, which emerged in Turkey between the years 2006-2015, their numbers and area distributions are shown in Table 1. With the examination of Table 1, it is possible the state that among the period of 2006-2015; the year 2013 reached the highest level in terms of the number of forest fires $(16.1 \%)$, yet the year 2008 reached highest value relating to amount of burnt area (33.4\%). The reason of why the year 2013 has the highest number of fire, is explained by the social events such as communal violence occurred in Turkey during 2013. It is also emerged that, with respect to amount of burnt area, the year 2008 has the highest value. This situation can be explained with the fact that the temperatures of 2008 were $0.5-2.4{ }^{\circ} \mathrm{C}$ 
higher than the average seasonal normals (1971-2000) as shown in Figure 4. This also causes the increase in number and burnt areas by means of forest fire. Moreover, it is obvious that our findings are supported by Kucuk and Saglam (2004).

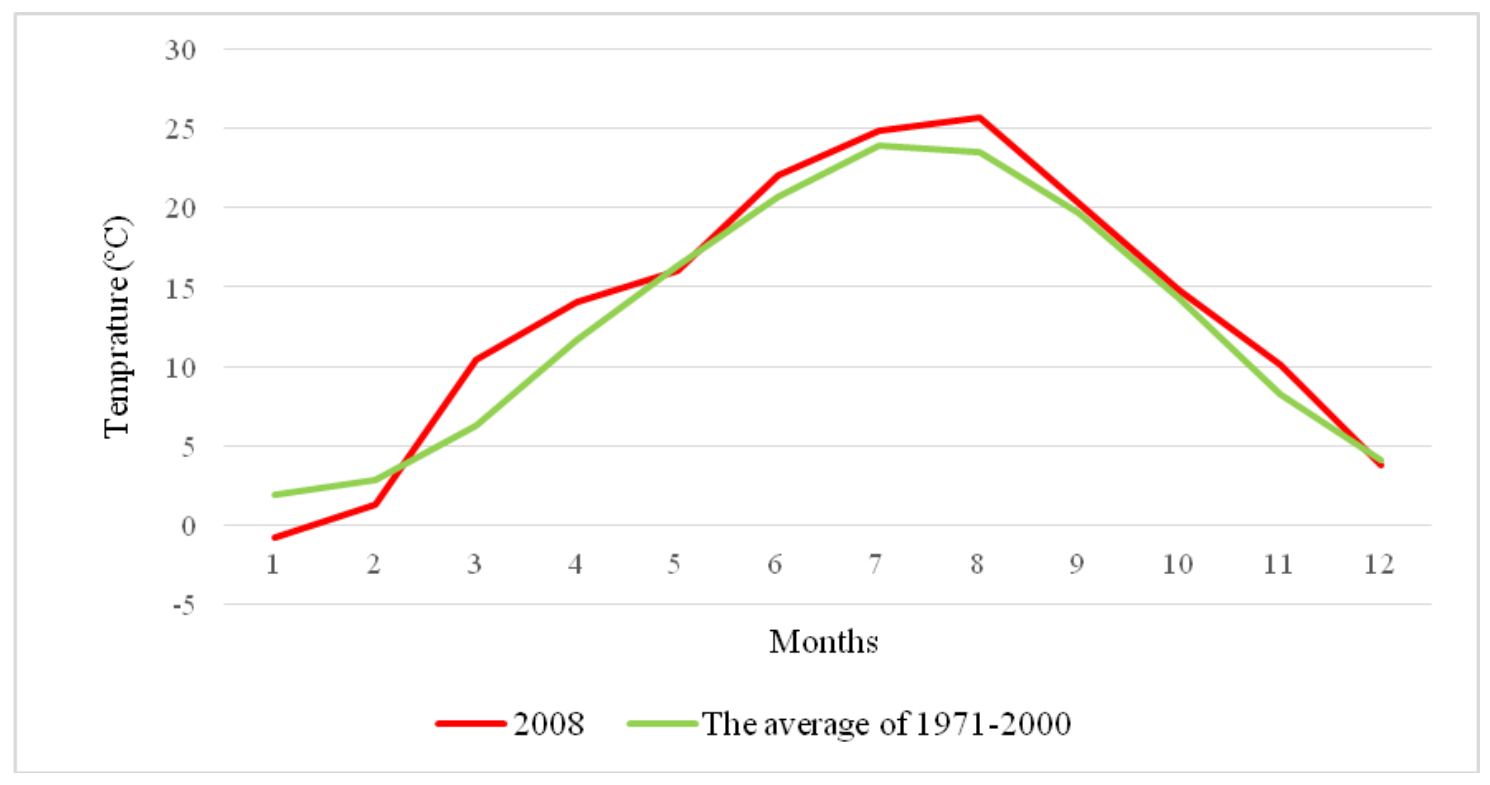

Figure 4. Average monthly temperatures of 1971-2000 and 2008 (MGM, 2017)

In Turkey, the main causes of the forest fires are human being (99\%) and lightning (1\%) (Mol and Kucukosmanoglu, 1998). According to Demir et al. (2009), forest fires have many causes in Turkey, such as; human activities (smoking, negligence, carelessness, accidents, deliberate fire setting etc.), shortage of basic equipment for fire fighting, climate and weather variation (lightning etc.) and unknown reasons.

Among the causes of forest fires, four main subjects; Intentional, NegligenceAccident, Natural (Lightning) and Unknown are particularly chosen and their values by years calculated in detail. In Table 1, it is important to point out that during the year 2008; number value of Intentional is $18.1 \%$, field value of Negligence-Accident is $43.7 \%$ and field value of Natural (Lightning) is 29.8\%. Furthermore, during the year 2013 ; it is possible to find out that by $24.4 \%$ in terms of number value and by $30.7 \%$ in terms of field value, Unknown has reached its highest values. During the examination period (2006-2015), within the range of these causes, in respect to number value Intentional and in accordance to its field value, Negligence-Accident comes forward.

For this reason, regarding the protection of forests from fires; more emphasis should be put on to the issues of public awareness and education. These findings and suggestions of ours are also validated by related work of Ryan (2012).

Detailed examination through Table 2 makes possible to reveal total forest fires within the mentioned years, in terms of number and area. For the year 2008, number value of fires generated from Intentional causes calculated as $1.6 \%$ and for the year 2007 field value is found as $1.9 \%$. In addition to this, it is revealed through Table 2 that in 2013 number value of Negligence-Accident obtained as $6.1 \%$ and in 2008 field value as $29.5 \%$. In 2007, number value of Natural (Lightning) is obtained as $1.7 \%$ and in 2008 field value as $0.8 \%$. Finally, in 2013 with respect to number value of Unknown as $7.8 \%$ and field value as 6.5\%; all of these mentioned years have reached their highest rates 
within Table 2. It seems clear that, likewise in Table 1, the year 2008 and the year 2013 comes forward in also Table 2. On the other hand, within mentioned decade when the total causes of forest fires are examined; it is seen that the Negligence-Accident has the first place concerning both number value $(46.1 \%)$ and area value $(67.6 \%)$. This finding shows that, fires are mostly caused by negligence and accident, rather than natural or unknown ones among the reasons of forest fires. For this reason, in order to protect forests from fire before the fire starts, precautions such as; fire safety roads, firebreaks, transportation roads, silvicultural measures, arrangement of flammable materials etc. are should be given priority and they should be practiced accurately.

In our work, Tables 3 and 4 show the field distribution of forest fires with respect to regional forest directorates. Table 3 reveals the field distribution, while Table 4 shows the numerical distribution.

As it is understood by generating and analyzing Tables 3 and 4, related to the regional forest directorates and field distribution of forest fires in terms of number and field (hectare) between the years 2006 and 2015 it is seen that numerical findings of Izmir, Antalya and Mugla reach the highest rates. Therefore it is possible to state that forest fires are mainly concentrated upon these three regions in Turkey. As being sensitive to forest fires; this is one of the reasons why Izmir is selected as our study subject.

It is also seen that our findings regarding to these observations are supported by the research that carried out by Mol and Kucukosmanoglu (1997) and Kucuk and Unal (2011).

\section{Forest fire statistics in İzir}

In Izmir there are many factors that destroy the forests. Among these destruction factors besides grazing, smuggling, insect damages and breeding, fire is the most dangerous one which especially harms plant species. All these mentioned factors are effective in Izmir Regional Directorate of Forestry that located in the west of Turkey as our study area.

Akyuz and Kucukosmanoglu (1997) especially draw attention to the fact that fire is the most important reason leading to destruction of forest resources in Izmir.

Izmir Regional Directorate of Forestry has the total area of 248986 ha. Approximately 968078 ha that constitutes the $39 \%$ of this area is covered with forests (GDF, 2016). Bayındır, Bergama, Izmir, Menderes, Akhisar, Demirci, Gördes, and Manisa are the names of the eight enterprises that Izmir Regional Directorate of Forestry contains. Between the years 2006-2015, number distribution of forest fires with respect to these State Forest Enterprises are stated and analyzed in Table 5. Within this 10 years period, calculations carried out in Table 5 expose that total fire number is 2333 and average fire number is 233. Also it is indicated in Table 6 that amount of burnt area is 7963 ha and the average amount of burnt area is calculated as 796 ha during this 10 years. As can be understood through the detailed investigation of Tables 5 and 6 , Izmir Forest Enterprise steps forward with highest values in respect to area and number, concerning the forest fires. This situation reveals similar results that forest fires reach the highest values of both number and field value, as we obtained from the examination of Izmir Regional Directorate of Forestry (Tables 3 and 4). These relevant findings indicate that Izmir Region has a sensitive status concerning the forest fires. Therefore; during the fire seasons, it is a necessity to take all the required precautions against fires in Izmir. 
Table 1. Forest fires in Turkey between 2006 and 2015

\begin{tabular}{|c|c|c|c|c|c|c|c|c|c|c|c|c|c|c|c|c|c|c|c|c|}
\hline \multirow{3}{*}{ Year } & \multirow{2}{*}{\multicolumn{2}{|c|}{$\begin{array}{l}\text { Numbers of forest } \\
\text { fires }\end{array}$}} & \multirow{2}{*}{\multicolumn{2}{|c|}{$\begin{array}{l}\text { Amount of } \\
\text { burnt area }\end{array}$}} & \multicolumn{16}{|c|}{ Causes of forest fires } \\
\hline & & & & & \multicolumn{4}{|c|}{ Intentional } & \multicolumn{4}{|c|}{ Negligence-accident } & \multicolumn{4}{|c|}{\begin{tabular}{|l|} 
Natural (lightning) \\
\end{tabular}} & \multicolumn{4}{|c|}{ Unknown } \\
\hline & Number & $\begin{array}{c}\text { Number } \\
\%\end{array}$ & $\begin{array}{c}\text { Field } \\
\text { (ha) }\end{array}$ & $\begin{array}{c}\text { Field } \\
(\%)\end{array}$ & Number & $\begin{array}{c}\text { Number } \\
\%\end{array}$ & $\begin{array}{c}\text { Field } \\
\text { (ha) }\end{array}$ & $\begin{array}{c}\text { Field } \\
(\%)\end{array}$ & Number & $\begin{array}{c}\text { Number } \\
\%\end{array}$ & $\begin{array}{c}\text { Field } \\
\text { (ha) }\end{array}$ & \begin{tabular}{|c|} 
Field \\
$(\%)$
\end{tabular} & Number & $\begin{array}{c}\text { Number } \\
\%\end{array}$ & $\begin{array}{c}\text { Field } \\
\text { (ha) }\end{array}$ & \begin{tabular}{|c|} 
Field \\
$(\%)$
\end{tabular} & Number & $\begin{array}{c}\text { Number } \\
\%\end{array}$ & Field (ha) & $\begin{array}{c}\text { Field } \\
(\%)\end{array}$ \\
\hline 2006 & 2227 & 9.6 & 7762 & 8.7 & 166 & 8 & 206 & 2.7 & 1315 & 12.2 & 5873 & 9.8 & 330 & 10.9 & 543 & 23.2 & 416 & 5.6 & 1139 & 6 \\
\hline 2007 & 2829 & 12.1 & 11664 & 13.1 & 292 & 14 & 1705 & 22.3 & 1642 & 15.3 & 7994 & 13.3 & 407 & 13.4 & 243 & 10.4 & 488 & 6.5 & 1722 & 9.1 \\
\hline 2008 & 2135 & 9.2 & 29749 & 33.4 & 377 & 18.1 & 797 & 10.4 & 1018 & 9.5 & 26283 & 43.7 & 330 & 10.9 & 699 & 29.8 & 410 & 5.5 & 1970 & 10.4 \\
\hline 2009 & 1793 & 7.7 & 4679 & 5.3 & 231 & 11.1 & 792 & 10.3 & 884 & 8.2 & 3082 & 5.1 & 333 & 11 & 105 & 4.5 & 345 & 4.6 & 700 & 3.7 \\
\hline 2010 & 1861 & 8.0 & 3317 & 3.7 & 146 & 7 & 526 & 6.9 & 861 & 8 & 1851 & 3.1 & 281 & 9.3 & 69 & 2.9 & 573 & 7.7 & 871 & 4.6 \\
\hline 2011 & 1954 & 8.4 & 3612 & 4.1 & 153 & 7.3 & 283 & 3.7 & 1067 & 9.9 & 2368 & 3.9 & 130 & 4.3 & 39 & 1.7 & 604 & 8.1 & 922 & 4.9 \\
\hline 2012 & 2450 & 10.5 & 10454 & 11.7 & 197 & 9.4 & 1615 & 21.1 & 936 & 8.7 & 5780 & 9.6 & 373 & 12.3 & 334 & 14.3 & 944 & 12.7 & 2725 & 14.4 \\
\hline 2013 & 3755 & 16.1 & 11456 & 12.9 & 260 & 12.5 & 1478 & 19.3 & 1419 & 13.2 & 4051 & 6.7 & 258 & 8.5 & 138 & 5.9 & 1818 & 24.4 & 5789 & 30.7 \\
\hline 2014 & 2149 & 9.2 & 3117 & 3.5 & 127 & 6.1 & 85 & 1.1 & 801 & 7.5 & 1682 & 2.8 & 328 & 10.8 & 77 & 3.3 & 893 & 12 & 1273 & 6.7 \\
\hline 2015 & 2150 & 9.2 & 3219 & 3.6 & 138 & 6.6 & 167 & 2.2 & 794 & 7.4 & 1198 & 2 & 257 & 8.5 & 95 & 4.1 & 961 & 12.9 & 1759 & 9.3 \\
\hline Total & 23303 & 100 & 89029 & 100 & 2087 & 100 & 7654 & 100 & 10737 & 100 & 60162 & 100 & 3027 & 100 & 2342 & 100 & 7452 & 100 & 18870 & 100 \\
\hline
\end{tabular}

Table 2. Evaluation of the total forest fires in terms of number and area between the years 2006 and 2015

\begin{tabular}{|c|c|c|c|c|c|c|c|c|c|c|c|c|c|c|c|c|c|c|}
\hline \multirow{3}{*}{ Year } & \multirow{3}{*}{\begin{tabular}{|c|}
$\begin{array}{c}\text { Numbers of } \\
\text { forest fires }\end{array}$ \\
Number \\
\end{tabular}} & \multirow{3}{*}{$\begin{array}{c}\begin{array}{c}\text { Amount of } \\
\text { burnt area }\end{array} \\
\begin{array}{c}\text { Field } \\
\text { (ha) }\end{array} \\
\end{array}$} & \multicolumn{16}{|c|}{\begin{tabular}{|l} 
Causes of forest fires \\
\end{tabular}} \\
\hline & & & \multicolumn{4}{|c|}{ Intentional } & \multicolumn{4}{|c|}{ Negligence-accident } & \multicolumn{4}{|c|}{ Natural (lightning) } & \multicolumn{4}{|c|}{ Unknown } \\
\hline & & & Number & $\underset{\%}{\text { Number }}$ & $\begin{array}{c}\text { Field } \\
\text { (ha) }\end{array}$ & $\begin{array}{c}\text { Field } \\
(\%)\end{array}$ & Number & $\underset{\%}{\text { Number }}$ & $\begin{array}{c}\text { Field } \\
\text { (ha) }\end{array}$ & $\begin{array}{c}\text { Field } \\
(\%)\end{array}$ & Number & $\underset{\%}{\text { Number }}$ & $\begin{array}{c}\text { Field } \\
\text { (ha) }\end{array}$ & $\begin{array}{c}\text { Field } \\
(\%)\end{array}$ & Number & $\underset{\%}{\text { Number }}$ & $\begin{array}{c}\text { Field } \\
\text { (ha) }\end{array}$ & $\begin{array}{c}\text { Field } \\
(\%)\end{array}$ \\
\hline 2006 & 2227 & 7762 & 166 & 0.7 & 206 & 0.2 & 1315 & 5.6 & 5873 & 6.6 & 330 & 1.4 & 543 & 0.6 & 416 & 1.8 & 1139 & 1.3 \\
\hline 2007 & 2829 & 11664 & 292 & 1.3 & 1705 & 1.9 & 1642 & 7 & 7994 & 9 & 407 & 1.7 & 243 & 0.3 & 488 & 2.1 & 1722 & 1.9 \\
\hline 2008 & 2135 & 29749 & 377 & 1.6 & 797 & 0.9 & 1018 & 4.4 & 26283 & 29.5 & 330 & 1.4 & 699 & 0.8 & 410 & 1.8 & 1970 & 2.2 \\
\hline 2009 & 1793 & 4679 & 231 & 1 & 792 & 0.9 & 884 & 3.8 & 3082 & 3.5 & 333 & 1.4 & 105 & 0.1 & 345 & 1.5 & 700 & 0.8 \\
\hline 2010 & 1861 & 3317 & 146 & 0.6 & 526 & 0.6 & 861 & 3.7 & 1851 & 2.1 & 281 & 1.2 & 69 & 0.1 & 573 & 2.5 & 871 & 1 \\
\hline 2011 & 1954 & 3612 & 153 & 0.7 & 283 & 0.3 & 1067 & 4.6 & 2368 & 2.7 & 130 & 0.6 & 39 & 0.1 & 604 & 2.6 & 922 & 1 \\
\hline 2012 & 2450 & 10454 & 197 & 0.8 & 1615 & 1.8 & 936 & 4 & 5780 & 6.5 & 373 & 1.6 & 334 & 0.4 & 944 & 4.1 & 2725 & 3.1 \\
\hline 2013 & 3755 & 11456 & 260 & 1.1 & 1478 & 1.7 & 1419 & 6.1 & 4051 & 4.6 & 258 & 1.1 & 138 & 0.2 & 1818 & 7.8 & 5789 & 6.5 \\
\hline 2014 & 2149 & 3117 & 127 & 0.5 & 85 & 0.1 & 801 & 3.4 & 1682 & 1.9 & 328 & 1.4 & 77 & 0.1 & 893 & 3.8 & 1273 & 1.4 \\
\hline 2015 & 2150 & 3219 & 138 & 0.6 & 167 & 0.2 & 794 & 3.4 & 1198 & 1.3 & 257 & 1.1 & 95 & 0.1 & 961 & 4.1 & 1759 & 2 \\
\hline Total & 23303 & 89029 & 2087 & 9 & 7654 & 8.6 & 10737 & 46.1 & 60162 & 67.6 & 3027 & 13 & 2342 & 2.6 & 7452 & 32 & 18870 & 21.2 \\
\hline
\end{tabular}


Table 3. The distribution of the field (ha) to the regional directorates of forest fires between 2006 and 2015

\begin{tabular}{|c|c|c|c|c|c|c|c|c|c|c|c|c|c|c|c|c|c|c|c|c|}
\hline \multirow{2}{*}{$\begin{array}{c}\text { Regional } \\
\text { directorates } \\
\text { of forestry }\end{array}$} & \multicolumn{2}{|c|}{2006} & \multicolumn{2}{|c|}{2007} & \multicolumn{2}{|c|}{2008} & \multicolumn{2}{|c|}{2009} & \multicolumn{2}{|c|}{2010} & \multicolumn{2}{|c|}{2011} & \multicolumn{2}{|c|}{2012} & \multicolumn{2}{|c|}{2013} & \multicolumn{2}{|c|}{2014} & \multicolumn{2}{|c|}{2015} \\
\hline & $\begin{array}{c}\text { Field } \\
\text { (ha) }\end{array}$ & $\begin{array}{c}\text { Field } \\
(\%)\end{array}$ & $\begin{array}{c}\text { Field } \\
\text { (ha) }\end{array}$ & $\begin{array}{c}\text { Field } \\
(\%)\end{array}$ & $\begin{array}{c}\text { Field } \\
\text { (ha) }\end{array}$ & $\begin{array}{c}\text { Field } \\
(\%)\end{array}$ & $\begin{array}{c}\text { Field } \\
\text { (ha) }\end{array}$ & $\begin{array}{c}\text { Field } \\
(\%)\end{array}$ & $\begin{array}{c}\text { Field } \\
\text { (ha) }\end{array}$ & $\begin{array}{c}\text { Field } \\
(\%)\end{array}$ & $\begin{array}{c}\text { Field } \\
\text { (ha) }\end{array}$ & $\begin{array}{c}\text { Field } \\
(\%)\end{array}$ & $\begin{array}{c}\text { Field } \\
\text { (ha) }\end{array}$ & $\begin{array}{c}\text { Field } \\
(\%)\end{array}$ & $\begin{array}{c}\text { Field } \\
\text { (ha) }\end{array}$ & $\begin{array}{c}\text { Field } \\
(\%)\end{array}$ & $\begin{array}{r}\text { Field } \\
\text { (ha) }\end{array}$ & $\begin{array}{c}\text { Field } \\
(\%)\end{array}$ & $\begin{array}{c}\text { Field } \\
\text { (ha) }\end{array}$ & $\begin{array}{c}\text { Field } \\
(\%)\end{array}$ \\
\hline Adana & 443 & 5.7 & 704 & 6 & 415 & 1.4 & 183 & 3.9 & 237 & 7.1 & 222 & 6.1 & 916 & 8.8 & 875 & 7.6 & 145 & 4.6 & 402 & 12.5 \\
\hline Amasya & 93 & 1.2 & 413 & 3.5 & 46 & 0.2 & 85 & 1.8 & 306 & 9.2 & 158 & 4.4 & 139 & 1.3 & 280 & 2.4 & 201 & 6.4 & 119 & 3.7 \\
\hline Ankara & 314 & 4 & 80 & 0.7 & 63 & 0.2 & 65 & 1.4 & 43 & 1.3 & 165 & 4.6 & 158 & 1.5 & 177 & 1.5 & 36 & 1.2 & 15 & 0.5 \\
\hline Antalya & 515 & 6.6 & 2093 & 17.9 & 17026 & 57.2 & 469 & 10 & 503 & 15.2 & 92 & 2.5 & 653 & 6.2 & 1312 & 11.5 & 234 & 7.5 & 197 & 6.1 \\
\hline Artvin & 7 & 0.1 & 6 & 0.1 & 34 & 0.1 & - & 0 & 42 & 1.3 & 20 & 0.6 & 7 & 0.1 & 20 & 0.2 & 9 & 0.3 & 5 & 0.2 \\
\hline Balıkesir & 302 & 3.9 & 543 & 4.7 & 1974 & 6.6 & 328 & 7 & 64 & 1.9 & 421 & 11.7 & 658 & 6.3 & 2350 & 20.5 & 33 & 1.1 & 39 & 1.2 \\
\hline Bolu & 284 & 3.7 & 80 & 0.7 & 29 & 0.1 & 12 & 0.3 & 85 & 2.6 & 83 & 2.3 & 65 & 0.6 & 77 & 0.7 & 14 & 0.4 & 51 & 1.6 \\
\hline Bursa & 159 & 2 & 386 & 3.3 & 54 & 0.2 & 452 & 9.7 & 108 & 3.3 & 121 & 3.4 & 343 & 3.3 & 532 & 4.6 & 40 & 1.3 & 263 & 8.2 \\
\hline Denizli & 61 & 0.8 & 369 & 3.2 & 71 & 0.2 & 88 & 1.9 & 91 & 2.7 & 116 & 3.2 & 235 & 2.2 & 122 & 1.1 & 80 & 2.6 & 34 & 1.1 \\
\hline Elazığ & 104 & 1.3 & 338 & 2.9 & 859 & 2.9 & 210 & 4.5 & 235 & 7.1 & 73 & 2 & 20 & 0.2 & 53 & 0.5 & 63 & 2 & 71 & 2.2 \\
\hline Erzurum & 65 & 0.8 & 28 & 0.2 & 14 & 0 & - & 0 & - & 0 & 40 & 1.1 & 66 & 0.6 & 55 & 0.5 & 9 & 0.3 & 83 & 2.6 \\
\hline Eskişehir & 66 & 0.9 & 107 & 0.9 & 24 & 0.1 & 70 & 1.5 & 46 & 1.4 & 54 & 1.5 & 105 & 1 & 155 & 1.4 & 22 & 0.7 & 5 & 0.2 \\
\hline Giresun & 10 & 0.1 & 33 & 0.3 & 51 & 0.2 & 11 & 0.2 & 106 & 3.2 & 27 & 0.7 & 32 & 0.3 & 91 & 0.8 & 89 & 2.9 & 74 & 2.3 \\
\hline Isparta & 49 & 0.6 & 55 & 0.5 & 61 & 0.2 & 38 & 0.8 & 130 & 3.9 & 127 & 3.5 & 297 & 2.8 & 97 & 0.8 & 61 & 2 & 54 & 1.7 \\
\hline İstanbul & 67 & 0.9 & 263 & 2.3 & 96 & 0.3 & 90 & 1.9 & 8 & 0.2 & 67 & 1.9 & 107 & 1 & 77 & 0.7 & 18 & 0.6 & 41 & 1.3 \\
\hline İzmir & 579 & 7.5 & 963 & 8.3 & 1790 & 6 & 1603 & 34.3 & 502 & 15.1 & 733 & 20.3 & 474 & 4.5 & 862 & 7.5 & 270 & 8.7 & 153 & 4.8 \\
\hline K. Maraş & 45 & 0.6 & 949 & 8.1 & 710 & 2.4 & 78 & 1.7 & 162 & 4.9 & 204 & 5.7 & 3669 & 35.1 & 1579 & 13.8 & 160 & 5.1 & 184 & 5.7 \\
\hline Kastamonu & 166 & 2.1 & 311 & 2.7 & 83 & 0.3 & 26 & 0.6 & 48 & 1.4 & 40 & 1.1 & 205 & 2 & 77 & 0.7 & 46 & 1.5 & 30 & 0.9 \\
\hline Kayseri & - & 0 & - & 0 & - & 0 & - & 0 & - & 0 & 161 & 4.5 & 51 & 0.5 & 203 & 1.8 & 69 & 2.2 & 41 & 1.3 \\
\hline Konya & 132 & 1.7 & 42 & 0.4 & 17 & 0.1 & 88 & 1.9 & 106 & 3.2 & 78 & 2.2 & 85 & 0.8 & 182 & 1.6 & 49 & 1.6 & 64 & 2 \\
\hline Kütahya & 574 & 7.4 & 581 & 5 & 15 & 0.1 & 25 & 0.5 & 11 & 0.3 & 14 & 0.4 & 183 & 1.8 & 72 & 0.6 & 52 & 1.7 & 6 & 0.2 \\
\hline Mersin & 29 & 0.4 & 1053 & 9 & 5080 & 17.1 & 80 & 1.7 & 105 & 3.2 & 114 & 3.2 & 505 & 4.8 & 509 & 4.4 & 79 & 2.5 & 282 & 8.8 \\
\hline Muğla & 3416 & 44 & 1531 & 13.1 & 665 & 2.2 & 260 & 5.6 & 160 & 4.8 & 165 & 4.6 & 242 & 2.3 & 972 & 8.5 & 724 & 23.2 & 184 & 5.7 \\
\hline Sakarya & 170 & 2.2 & 321 & 2.8 & 192 & 0.6 & 358 & 7.7 & 87 & 2.6 & 106 & 2.9 & 99 & 0.9 & 117 & 1 & 28 & 0.9 & 34 & 1.1 \\
\hline Şanliurfa & - & 0 & - & 0 & - & 0 & - & 0 & - & 0 & 45 & 1.2 & 89 & 0.9 & 387 & 3.4 & 302 & 9.7 & 536 & 16.7 \\
\hline Trabzon & 10 & 0.1 & 40 & 0.3 & 324 & 1.1 & 46 & 1 & 61 & 1.8 & 73 & 2 & 39 & 0.4 & 166 & 1.4 & 268 & 8.6 & 141 & 4.4 \\
\hline Zonguldak & 101 & 1.3 & 373 & 3.2 & 56 & 0.2 & 14 & 0.3 & 71 & 2.1 & 91 & 2.5 & 1013 & 9.7 & 57 & 0.5 & 19 & 0.6 & 111 & 3.4 \\
\hline Total & 7761 & 100 & 11662 & 100 & 29749 & 100 & 4679 & 100 & 3317 & 100 & 3610 & 100 & 10455 & 100 & 11456 & 100 & 3120 & 100 & 3219 & 100 \\
\hline
\end{tabular}

APPLIED ECOLOGY AND ENVIRONMENTAL RESEARCH 17(3): 6989-7001.

http://www.aloki.hu • ISSN 15891623 (Print) • ISSN 17850037 (Online)

DOI: http://dx.doi.org/10.15666/aeer/1703_69897001

(c) 2019, ALÖKI Kft., Budapest, Hungary 
Table 4. Numerical distribution (number) to the regional directorates of forest fires between 2006 and 2015

\begin{tabular}{|c|c|c|c|c|c|c|c|c|c|c|c|c|c|c|c|c|c|c|c|c|}
\hline \multirow{2}{*}{$\begin{array}{c}\text { Regional } \\
\text { directorates } \\
\text { of forestry }\end{array}$} & \multicolumn{2}{|c|}{2006} & \multicolumn{2}{|c|}{2007} & \multicolumn{2}{|c|}{2008} & \multicolumn{2}{|c|}{2009} & \multicolumn{2}{|c|}{2010} & \multicolumn{2}{|c|}{2011} & \multicolumn{2}{|l|}{2012} & \multicolumn{2}{|c|}{2013} & \multicolumn{2}{|c|}{2014} & \multicolumn{2}{|c|}{2015} \\
\hline & Number & $\%$ & Number & $\%$ & Number & $\%$ & Number & $\%$ & Number & $\%$ & Number & $\%$ & Number & $\%$ & Number & $\%$ & Number & $\%$ & Number & $\%$ \\
\hline Adana & 105 & 4.7 & 123 & 4.3 & 107 & 5 & 83 & 4.6 & 84 & 4.5 & 88 & 4.5 & 101 & 4.1 & 173 & 4.6 & 81 & 3.8 & 176 & 8.2 \\
\hline Amasya & 116 & 5.2 & 165 & 5.8 & 63 & 3 & 74 & 4.1 & 104 & 5.6 & 93 & 4.8 & 95 & 3.9 & 144 & 3.8 & 84 & 3.9 & 60 & 2.8 \\
\hline Ankara & 76 & 3.4 & 95 & 3.4 & 38 & 1.8 & 84 & 4.7 & 57 & 3.1 & 73 & 3.7 & 113 & 4.6 & 233 & 6.2 & 46 & 2.1 & 43 & 2 \\
\hline Antalya & 240 & 10.8 & 265 & 9.4 & 212 & 9.9 & 144 & 8 & 125 & 6.7 & 152 & 7.8 & 214 & 8.7 & 321 & 8.5 & 169 & 7.9 & 189 & 8.8 \\
\hline Artvin & 5 & 0.2 & 8 & 0.3 & 8 & 0.4 & - & 0 & 17 & 0.9 & 5 & 0.3 & 3 & 0.1 & 7 & 0.2 & 6 & 0.3 & 5 & 0.2 \\
\hline Balıkesir & 105 & 4.7 & 122 & 4.3 & 80 & 3.7 & 90 & 5 & 57 & 3.1 & 79 & 4 & 73 & 3 & 96 & 2.6 & 72 & 3.4 & 68 & 3.2 \\
\hline Bolu & 57 & 2.6 & 61 & 2.2 & 45 & 2.1 & 18 & 1 & 30 & 1.6 & 32 & 1.6 & 40 & 1.6 & 57 & 1.5 & 26 & 1.2 & 24 & 1.1 \\
\hline Bursa & 82 & 3.7 & 94 & 3.3 & 97 & 4.5 & 105 & 5.9 & 53 & 2.8 & 72 & 3.7 & 81 & 3.3 & 118 & 3.1 & 64 & 3 & 43 & 2 \\
\hline Denizli & 128 & 5.7 & 154 & 5.4 & 90 & 4.2 & 79 & 4.4 & 72 & 3.9 & 76 & 3.9 & 68 & 2.8 & 146 & 3.9 & 47 & 2.2 & 41 & 1.9 \\
\hline Elazı̆g & 27 & 1.2 & 56 & 2 & 77 & 3.6 & 45 & 2.5 & 47 & 2.5 & 14 & 0.7 & 4 & 0.2 & 72 & 1.9 & 79 & 3.7 & 84 & 3.9 \\
\hline Erzurum & 26 & 1.2 & 8 & 0.3 & 8 & 0.4 & 2 & 0.1 & 1 & 0.1 & 7 & 0.4 & 16 & 0.7 & 21 & 0.6 & 5 & 0.2 & 17 & 0.8 \\
\hline Eskişehir & 43 & 1.9 & 59 & 2.1 & 42 & 2 & 51 & 2.8 & 44 & 2.4 & 32 & 1.6 & 38 & 1.6 & 79 & 2.1 & 21 & 1 & 12 & 0.6 \\
\hline Giresun & 11 & 0.5 & 19 & 0.7 & 27 & 1.3 & 9 & 0.5 & 60 & 3.2 & 16 & 0.8 & 30 & 1.2 & 40 & 1.1 & 46 & 2.1 & 43 & 2 \\
\hline Isparta & 47 & 2.1 & 53 & 1.9 & 69 & 3.2 & 76 & 4.2 & 78 & 4.2 & 56 & 2.9 & 105 & 4.3 & 120 & 3.2 & 70 & 3.3 & 74 & 3.4 \\
\hline İstanbul & 151 & 6.8 & 186 & 6.6 & 136 & 6.4 & 115 & 6.4 & 42 & 2.3 & 172 & 8.8 & 228 & 9.3 & 271 & 7.2 & 108 & 5 & 153 & 7.1 \\
\hline İzmir & 179 & 8 & 256 & 9 & 151 & 7.1 & 183 & 10.2 & 216 & 11.6 & 197 & 10.1 & 269 & 11 & 344 & 9.2 & 284 & 13.2 & 265 & 12.3 \\
\hline K. Maraş & 71 & 3.2 & 100 & 3.5 & 64 & 3 & 79 & 4.4 & 119 & 6.4 & 78 & 4 & 132 & 5.4 & 262 & 7 & 155 & 7.2 & 170 & 7.9 \\
\hline Kastamonu & 97 & 4.4 & 169 & 6 & 67 & 3.1 & 39 & 2.2 & 48 & 2.6 & 63 & 3.2 & 120 & 4.9 & 146 & 3.9 & 88 & 4.1 & 57 & 2.7 \\
\hline Kayseri & - & 0 & - & 0 & - & 0 & - & 0 & - & 0 & 54 & 2.8 & 16 & 0.7 & 69 & 1.8 & 27 & 1.3 & 26 & 1.2 \\
\hline Konya & 42 & 1.9 & 37 & 1.3 & 30 & 1.4 & 36 & 2 & 51 & 2.7 & 32 & 1.6 & 50 & 2 & 91 & 2.4 & 31 & 1.4 & 29 & 1.3 \\
\hline Kütahya & 46 & 2.1 & 63 & 2.2 & 48 & 2.2 & 55 & 3.1 & 34 & 1.8 & 41 & 2.1 & 54 & 2.2 & 90 & 2.4 & 55 & 2.6 & 33 & 1.5 \\
\hline Mersin & 50 & 2.2 & 128 & 4.5 & 93 & 4.4 & 60 & 3.3 & 90 & 4.8 & 98 & 5 & 75 & 3.1 & 155 & 4.1 & 91 & 4.2 & 80 & 3.7 \\
\hline Muğla & 326 & 14.6 & 415 & 14.7 & 348 & 16.3 & 252 & 14.1 & 309 & 16.6 & 267 & 13.7 & 383 & 15.6 & 395 & 10.5 & 320 & 14.9 & 254 & 11.8 \\
\hline Sakarya & 57 & 2.6 & 80 & 2.8 & 98 & 4.6 & 74 & 4.1 & 54 & 2.9 & 58 & 3 & 56 & 2.3 & 78 & 2.1 & 33 & 1.5 & 46 & 2.1 \\
\hline Şanlıurfa & - & 0 & - & 0 & - & 0 & - & 0 & - & 0 & 21 & 1.1 & 23 & 0.9 & 101 & 2.7 & 66 & 3.1 & 89 & 4.1 \\
\hline Trabzon & 3 & 0.1 & 5 & 0.2 & 26 & 1.2 & 9 & 0.5 & 25 & 1.3 & 21 & 1.1 & 6 & 0.2 & 41 & 1.1 & 29 & 1.3 & 22 & 1 \\
\hline Zonguldak & 137 & 6.2 & 108 & 3.8 & 111 & 5.2 & 30 & 1.7 & 44 & 2.4 & 57 & 2.9 & 57 & 2.3 & 85 & 2.3 & 46 & 2.1 & 47 & 2.2 \\
\hline Total & 2227 & 100 & 2829 & 100 & 2135 & 100 & 1792 & 100 & 1861 & 100 & 1954 & 100 & 2450 & 100 & 3755 & 100 & 2149 & 100 & 2150 & 100 \\
\hline
\end{tabular}


Table 5. Distribution of forest fire numbers to the State Forest Enterprises in the İzmir Regional Directorates between 2006 and 2015

\begin{tabular}{|c|c|c|c|c|c|c|c|c|c|c|c|c|c|c|c|c|c|c|c|c|c|c|c|c|}
\hline \multirow[t]{2}{*}{$\begin{array}{c}\text { State Forest } \\
\text { Enterprise }\end{array}$} & \multicolumn{2}{|l|}{2006} & \multicolumn{2}{|c|}{2007} & \multicolumn{2}{|c|}{2008} & \multicolumn{2}{|l|}{2009} & \multicolumn{2}{|c|}{2010} & \multicolumn{2}{|l|}{2011} & \multicolumn{2}{|c|}{2012} & \multicolumn{2}{|l|}{2013} & \multicolumn{2}{|l|}{2014} & \multicolumn{2}{|l|}{2015} & \multicolumn{2}{|c|}{10 years total } & \multicolumn{2}{|c|}{$\begin{array}{l}\text { 10-year } \\
\text { average }\end{array}$} \\
\hline & Number & $\%$ & Number & $\%$ & Number & $\%$ & Number & $\%$ & Number & $\%$ & Number & $\%$ & Number & $\%$ & Number & $\%$ & Number & $\%$ & Number & $\%$ & Number & $\%$ & Number & $\%$ \\
\hline Bayındır & 24 & 13.4 & 26 & 10.2 & 15 & 9.9 & 30 & 16.4 & 13 & 6.2 & 25 & 13 & 29 & 10.9 & 24 & 7 & 18 & 6.3 & 28 & 10.6 & 232 & 9.9 & 23 & 9.9 \\
\hline Bergama & 19 & 10.6 & 17 & 6.6 & 23 & 15.1 & 21 & 11.5 & 19 & 9 & 26 & 13.5 & 44 & 16.5 & 45 & 13 & 19 & 6.7 & 44 & 16.6 & 277 & 11.9 & 28 & 12 \\
\hline İzmir & 53 & 29.6 & 56 & 21.9 & 33 & 21.7 & 48 & 26.2 & 71 & 33.8 & 71 & 36.8 & 54 & 20.3 & 85 & 24.6 & 85 & 29.9 & 73 & 27.5 & 629 & 27 & 63 & 27 \\
\hline Menderes & & 0.0 & & 0 & & 0 & & 0 & & 0 & & 0 & 32 & 12 & 53 & 15.4 & 37 & 13 & 32 & 12.1 & 154 & 6.6 & 39 & 16.7 \\
\hline Akhisar & 31 & 17.3 & 55 & 21.5 & 42 & 27.6 & 28 & 15.3 & 46 & 21.9 & 32 & 16.6 & 49 & 18.4 & 67 & 19.4 & 43 & 15.1 & 53 & 20 & 446 & 19.1 & 45 & 19.3 \\
\hline Demirci & 12 & 6.7 & 30 & 11.7 & 8 & 5.3 & 17 & 9.3 & 16 & 7.6 & 7 & 3.6 & 15 & 5.6 & 19 & 5.5 & 22 & 7.7 & 6 & 2.3 & 152 & 6.5 & 15 & 6.4 \\
\hline Gördes & 13 & 7.3 & 19 & 7.4 & 4 & 2.6 & 1 & 0.5 & 11 & 5.2 & 2 & 1 & 10 & 3.8 & 5 & 1.4 & 17 & 6 & 7 & 2.6 & 89 & 3.8 & 9 & 3.9 \\
\hline Manisa & 27 & 15.1 & 53 & 20.7 & 27 & 17.8 & 38 & 20.8 & 34 & 16.2 & 30 & 15.5 & 33 & 12.4 & 47 & 13.6 & 43 & 15.1 & 22 & 8.3 & 354 & 15.2 & 35 & 15 \\
\hline Total & 179 & 100 & 256 & 100 & 152 & 100 & 183 & 100 & 210 & 100 & 193 & 100 & 266 & 100 & 345 & 100 & 284 & 100 & 265 & 100 & 2333 & 100 & 233 & 100 \\
\hline
\end{tabular}

Table 6. Amount of burnt area (ha) to the State Forest Enterprises in the İzmir Regional Directorates between 2006-2015

\begin{tabular}{|c|c|c|c|c|c|c|c|c|c|c|c|c|c|c|c|c|c|c|c|c|c|c|c|c|}
\hline \multirow{2}{*}{$\begin{array}{c}\text { State } \\
\text { Forest } \\
\text { Enterprise }\end{array}$} & \multicolumn{2}{|c|}{2006} & \multicolumn{2}{|c|}{2007} & \multicolumn{2}{|c|}{2008} & \multicolumn{2}{|c|}{2009} & \multicolumn{2}{|c|}{2010} & \multicolumn{2}{|c|}{2011} & \multicolumn{2}{|c|}{2012} & \multicolumn{2}{|c|}{2013} & \multicolumn{2}{|c|}{2014} & \multicolumn{2}{|c|}{2015} & \multicolumn{2}{|c|}{$\begin{array}{c}10 \text { years } \\
\text { total }\end{array}$} & \multicolumn{2}{|c|}{$\begin{array}{l}\text { 10-year } \\
\text { average }\end{array}$} \\
\hline & $\begin{array}{c}\text { Field } \\
\text { (ha) }\end{array}$ & $\%$ & $\begin{array}{c}\text { Field } \\
\text { (ha) }\end{array}$ & $\%$ & $\begin{array}{c}\text { Field } \\
\text { (ha) }\end{array}$ & $\%$ & $\begin{array}{c}\text { Field } \\
\text { (ha) }\end{array}$ & $\%$ & $\begin{array}{c}\text { Field } \\
\text { (ha) }\end{array}$ & $\%$ & $\begin{array}{c}\text { Field } \\
\text { (ha) }\end{array}$ & $\%$ & $\begin{array}{c}\text { Field } \\
\text { (ha) }\end{array}$ & $\%$ & $\begin{array}{c}\text { Field } \\
\text { (ha) }\end{array}$ & $\%$ & $\begin{array}{c}\text { Field } \\
\text { (ha) }\end{array}$ & $\%$ & $\begin{array}{c}\text { Field } \\
\text { (ha) }\end{array}$ & $\%$ & $\begin{array}{c}\text { Field } \\
\text { (ha) }\end{array}$ & $\%$ & $\begin{array}{c}\text { Field } \\
\text { (ha) }\end{array}$ & $\%$ \\
\hline Bayındır & 418 & 72.3 & 28 & 2.9 & 63 & 3.5 & 48 & 3 & 6 & 1.2 & 22 & 3 & 19 & 3.8 & 8 & 0.9 & 22 & 8.1 & 8 & 5.3 & 642 & 8.1 & 64 & 8 \\
\hline Bergama & 30 & 5.25 & 130 & 13.5 & 23 & 1.3 & 12 & 0.7 & 398 & 80.4 & 148 & 20.3 & 76 & 15.4 & 22 & 2.6 & 4 & 1.5 & 15 & 9.9 & 859 & 10.8 & 86 & 10.8 \\
\hline İzmir & 71 & 12.3 & 468 & 48.6 & 1513 & 84.5 & 1457 & 89.7 & 40 & 8.1 & 439 & 6.3 & 100 & 20.2 & 215 & 24.9 & 64 & 23.6 & 39 & 25.7 & 4407 & 55.3 & 441 & 55.4 \\
\hline Menderes & - & $\mathbf{0}$ & - & 0 & - & 0 & - & 0 & - & 0 & - & 0 & 94 & 19 & 57 & 6.6 & 26 & 9.6 & 11 & 7.2 & 188 & 2.4 & 47 & 5.9 \\
\hline Akhisar & 23 & 4 & 36 & 3.7 & 94 & 5.2 & 21 & 1.3 & 13 & 2.6 & 65 & 8.9 & 37 & 7.5 & 58 & 6.7 & 111 & 41 & 62 & 40.8 & 520 & 6.5 & 52 & 6.5 \\
\hline Demirci & 18 & 3.1 & 15 & 1.6 & 31 & 1.7 & 23 & 1.4 & 17 & 3.4 & 14 & 1.9 & 40 & 8.1 & 25 & 2.9 & 20 & 7.4 & 1 & 0.7 & 205 & 2.6 & 21 & 2.6 \\
\hline Gördes & 5 & 0.9 & 13 & 1.3 & 50 & 2.8 & 3 & 0.2 & 6 & 1.2 & 1 & 0.1 & 4 & 0.8 & 3 & 0.3 & 7 & 2.6 & 1 & 0.7 & 93 & 1.2 & 9 & 1.1 \\
\hline Manisa & 13 & 2.2 & 273 & 28.3 & 17 & 0.9 & 60 & 3.7 & 15 & 3 & 39 & 5.4 & 125 & 25.3 & 472 & 54.8 & 18 & 6.6 & 15 & 9.9 & 1048 & 13.2 & 105 & 13.2 \\
\hline Total & 578 & 100 & 963 & 100 & 1791 & 100 & 1625 & 100 & 495 & 100 & 728 & 100 & 495 & 100 & 862 & 100 & 271 & 100 & 152 & 100 & 7963 & 100 & 796 & 100 \\
\hline
\end{tabular}




\section{Turkish Forest Organization and present activities against fires}

Ministry of Agriculture and Forestry of Turkey and the Turkish General Directorate of Forestry have the main responsibility over the fire prevention and forest protection. All the organizational framework is set up in accordance with the Turkish Constitution (Dated 07.11.1982, Nr.2709, Section 169) and Turkish Forest Law (Dated 08.09.1956, Nr.6831, Section 68-76) besides relevant rules and regulations. The General Directorate of Forestry contains primary departments of fire control and forest protection.

General Directorate of the Turkish Forestry has three main sections which are: Forest Protection, Control of Forest Pests and Prevention and Control of Forest Fires. Especially, the section of Prevention and Control of Forest Fires is charged to prevent forest fires by means of the Regional forest directorates, forest enterprises and forest rangers. Fire suppression activities are realized by different kind of tools, equipment, man and machine powers and also sometimes by aerial supports which can be summarized as: fire attack crews, fire personnel, fire boss, fire lookouts, power chain saws, bulldozers, tractors, lorries, water trucks, pick-ups, off-road cars, water reservoirs, UHF and FM communication radios, telescopes, different masks, fire resistant clothes, chemical retardants, planes and helicopters. Furthermore; meteorological data is taken from the weather stations. Meantime various firebreaks, fuelbreaks, fire lookout roads and telephone lines are also constructed (Mol and Kucukosmanoglu, 1998).

\section{Discussion and conclusion}

Forest fires are one of the major problems in many countries. As we know, in context of location and climate properties; during fire seasons the environmental effects of drought, low rates of relative humidity and wind occur in Mediterranean, Aegean and Marmara Regions of Turkey. Due to these reasons; as being located at Aegean Region, Izmir Regional Directorate of Forestry is also at remarkable risk. However regarding the forest protection and fire prevention, there is no doubt that much development has been achieved in Turkey. Within the results of our study, fires are mostly caused by negligence and accident, rather than natural or unknown ones among the reasons of forest fires. During the examination period (2006-2015), within the range of these causes, in respect to number value Intentional and in accordance to its field value, Negligence-Accident comes forward.

For this reason, regarding the protection of forests from fires; more emphasis should be put on to the issues of public awareness and education. Between the years 2006 and 2015, it is seen that numerical findings of Izmir, Antalya and Muğla reach the highest rates. Therefore it is possible to state that forest fires are mainly concentrated upon these three regions in Turkey. Izmir Forest Enterprise steps forward with highest values in respect to area and number, concerning with forest fires.

There are 603 forest villages in İzmir. It is emphasized that the socio-economic level of the people which are living in or near the forest, is highly below the standard of living. According to statistical data, 343.513 forest villagers live there (GDF, 2016). The average income of forest villagers is $\$ 250$ per month, mainly from forestry work and farming Forest villagers regularly clear fields from forest land for subsistence farming. So, these villagers would like to use the forest without limitation for their survival. This brought out the forest-human activities relationship. Furthermore, this helps to explain why the three causes of forest fires are negligence, carelessness and accidents, unknown reasons, and deliberate fire setting. 
Demir et al. (2019) stated that however, with the fast-growing economy in Turkey, the present situation of forest fire protection is becoming severe. The main causes are that technical forest fire protection work has failed to keep up with social development and that regulation and management systems for forest fire protection are falling behind the present state of social development. Therefore, more effective regulations and rules should be set up soon, and a strategy should be developed in Turkey to carry out timely forest fire research work so as to cope with the new challenges of forest fire hazards in the future.

Boazountas et al. (2005) stated that most of the Mediterranean region suffers from extensive forest fires, and in some cases socioeconomic factors are key determinants in wildfire causality classification. Determinants are those components within the forestry environment that help explain the intensity and spread of wildfires. The module developed here incorporates five factors representing forestry profitability, demographic pressure, social tension, forestry culture, and organizational thinking. These factors are sufficient to explain the increase or decrease of fire risk. These factors can be modelled by considering variables which describe the behaviour of the socioeconomic fire risk (local permanent population, tourists, domestic animals, houses, type and height of vegetation) and constructing transformation functions that will determine the final value of fire risk.

Ayberk et al. (2010) has similar results as our research and stated that fire is a natural element and plays an important role in the natural dynamics of the forest ecosystems. However, fire can be a very destructive force. According to the establishment of new and modern fire prevention and suppression techniques, the number of fires and burning area will be getting decreased. Adverse burning conditions will occur in the future as they have been in the past. The important measures to be taken are firstly to keep away the fire from the forests and secondly to suppress the fire immediately and effectively as it is still at the beginning stage. The sooner a fire is detected and reported, the larger the initial attack force and the quicker it arrives at the fire, the more likely the fire will be contained at a small size. This could be realized by effective protective measures and well-trained fire fighting teams using appropriate high technology and equipment.

Therefore, all relevant findings in our study indicate that Izmir Region has a sensitive status concerning the forest fires. Therefore; during the fire seasons in Izmir, it is a necessity to take all the required measures against fires. Also, fire suppression activities in Izmir should be carried out by different kind of tools, equipment, man and machine powers and aerial supports such as fire attack crews, fire personnel, off-road cars, water reservoirs, UHF and FM communication radios, fire resistant clothes, planes and helicopters, etc. Due to the fact that Izmir Regional Directorate of Forestry where the forest fires are frequently occurred has a long fire season (5-8 months), flammable material reduction and road cleaning works in forests should be performed. Izmir Regional Directorate of Forestry is one of the most dangerous forest region directorate compared to other directorates in terms of both number of fires and burnt area. For this reason, in context of forest fires all essential precautions should be taken and applied carefully.

Acknowledgements. Authors would like to thank to Turkish General Directorate of Forestry and İzmir Directorate of Forestry. 


\section{REFERENCES}

[1] Akyuz, Y. F., Kucukosmanoglu, A. (1996): Studies on fire-damaged area in Turkey for GIS Application. - XVIII ISPRS-Congress, International Society for Phatogrammetry and Remote Sensing, Austria, 12-18 July, pp.45-49.

[2] Akyuz, Y. F., Kucukosmanoglu, A. (1997): Application of GIS for fire-damaged area in Izmir. - Proceedings of the XI World Forestry Congress, Turkey, 13-22 October, Vol. 1.

[3] Ayberk, H., Kucukosmanoglu, A., Cebeci, H. H. (2010): The structure and importance of fire suppressing organizations in Turkey. - Scientific Research and Essays 5: 456-460.

[4] Boazountas, M., Kallidromitou, D., Kassomenos, P. A., Passas, N. (2005): Forest fire risk analysis. - Human and Ecological Risk Assessment 11(3): 617-626.

[5] Bilgili, E., Kucuk, O. (2001): The importance of fire fuel in determination of fire sensitivity degrees. - 1st National Forestry Congress of Foresters Association of Turkey, 19-20 March, Turkey.

[6] Demir, M., Kucukosmanoglu, A., Hasdemir, M., Ozturk, T., Acar, H. (2009): Assessment of forest roads and firebreaks in Turkey. - African Journal of Biotechnology 18: 45534561.

[7] GDF (2016): Turkish General Directorate of Forestry. https://www.ogm.gov.tr/ekutuphane/Sayfalar/Istatistikler Forestry Statistics.

[8] Goltas, M., Demirel, T., Caglayan, I. (2017a): Visibility analysis of fire watchtowers using GIS. A case study in Dalaman State Forest Enterprise. - European Journal of Forest Engineering 3(2): 66-71.

[9] Goltas, M., Kucuk, O., Ayberk, H., Akkuzu, E. (2017b): Global Climate Change and Forest Fires. - In: Fakir, H. (ed.) International Symposium on New Horizons in Forestry. Proceedings \& Abstracts Book. Süleyman Demirel University, Isparta-Turkey.

[10] Kucuk, O., Saglam, B. (2004): Forest Fires and Fire Weather. - Kastamonu University, Journal of Forestry Faculty, Kastamonu 4(2): 220-230.

[11] Kucuk, O., Unal, S. (2011): Determination of fire sensitivity degree: a case study in Taşköprü State Forest Enterprise. - Artvin Çoruh University, Journal of Forestry Faculty 6(1): 28-34.

[12] Kucukosmanoglu, A. (1987): Classification of the Forest Fires and the Causes of Occurrence and Spread of Large Forest Fires in Turkey. - The Publication of General Directorate of Forestry, Ankara.

[13] MGM (2017): Turkish General Directorate of Meteorology. - https://www.mgm.gov.tr/.

[14] Mol, T., Kucukosmanoglu, A. (1998): Forest fires in Turkey. - Journal of Faculty of Forestry İstanbul Univertsity 48: 53-59.

[15] Mol, T., Kucukosmanoglu, A., Bilgili, E. (1997): Forest fires in global environment and changing attitudes toward fire. - Proceedings of the XI World Forestry Congress, Turkey, 13-22 October, Vol. 1, pp. 214-222.

[16] Noble, I. R., Gill, A. M., Bary, G. A. V. (1980): McArthur's fire-danger meters expressed as equations. - Australian Journal of Ecology 5(2): 201-203.

[17] Ryan, R. L. (2012): The influence of landscape preference and environmental education on public attitudes toward wildfire management in the Northeast Pine barrens (USA). Landscape and Urban Planning 107(1): 55-68.

[18] Uzmez, I. (2011): Forest Fire Control Activities and Their Efficiency in Turkey (A Case of Study of Izmir Forest Directorate). - Istanbul University Institute of Graduate Studies in Sciences, Istanbul. 